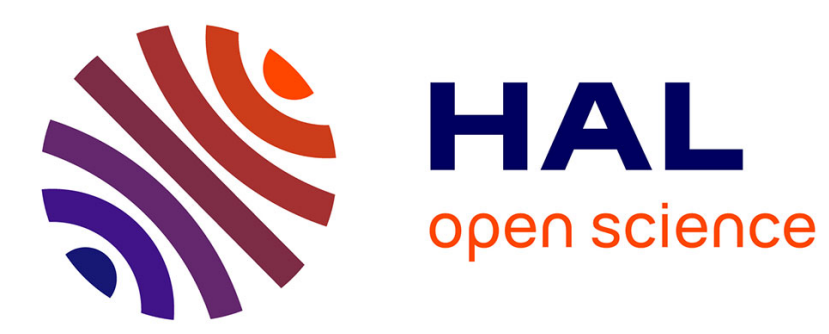

\title{
Capacity of wireless mesh networks: determining elements and insensible properties
}

Anis Ouni, Hervé Rivano, Fabrice Valois

\section{To cite this version:}

Anis Ouni, Hervé Rivano, Fabrice Valois. Capacity of wireless mesh networks: determining elements and insensible properties. [Research Report] RR-7171, INRIA. 2010. inria-00444891

\section{HAL Id: inria-00444891 \\ https://hal.inria.fr/inria-00444891}

Submitted on 7 Jan 2010

HAL is a multi-disciplinary open access archive for the deposit and dissemination of scientific research documents, whether they are published or not. The documents may come from teaching and research institutions in France or abroad, or from public or private research centers.
L'archive ouverte pluridisciplinaire HAL, est destinée au dépôt et à la diffusion de documents scientifiques de niveau recherche, publiés ou non, émanant des établissements d'enseignement et de recherche français ou étrangers, des laboratoires publics ou privés. 


\section{N R I A}

INSTITUT NATIONAL DE RECHERCHE EN INFORMATIQUE ET EN AUTOMATIQUE

Capacity of wireless mesh networks: determining elements and insensible properties

Anis Ouni — Hervé Rivano — Fabrice Valois

\section{$\mathbf{N}^{\circ} \mathbf{7 1 7 1}$}

January 2010

\section{apport}

de recherche 



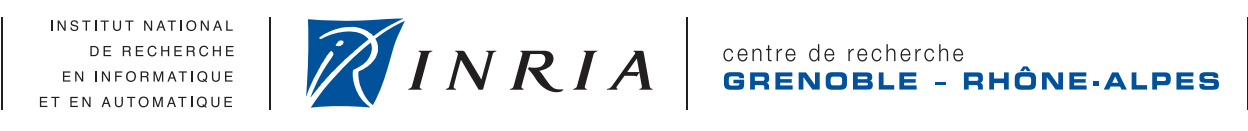

\title{
Capacity of wireless mesh networks: determining elements and insensible properties
}

\author{
Anis Ouni閳 Hervé Rivand $\mathbb{7}^{7}$, Fabrice Valois ${ }^{\dagger}$ \\ Thème : 1 \\ Équipes-Projets SWING \\ Rapport de recherche $\mathrm{n}^{\circ} 7171$ - January $2010-16$ pages
}

\begin{abstract}
The capacity of a multi-hop wireless network is the traffic payload that it can transport. This is a prominent quality of service issue, particularly in the highly constrained settings of 802.11 wireless mesh network. In this paper, we consider two complementary definitions of the capacity. A network-wise capacity is defined as the sum of the upload traffic, and a flow-wise capacity highlighting the unfairness among traffic flows. We study the behavior of these capacities face to several parameters: routing protocols, number and location of gateways bridging the network to the Internet, and the physical network topology. Thorough simulations highlight the insensitivity of the capacity face to these parameters while it is directly related to the congestion around the gateway. Furthermore, we show that if the number of gateways increases, the capacity tends to a maximum boundary.
\end{abstract}

Key-words: Wireless mesh network, network-wise capacity, flow-wise capacity, routing, network topology.

\footnotetext{
* Corresponding author - Partially supported by ANR VERSO ECOSCells

$\dagger$ Université de Lyon, INRIA, INSA Lyon, CITI, F-69621, France

$\ddagger$ CNRS-INRIA-Université de Nice-Sophia Antipolis Mascotte
}

Centre de recherche INRIA Grenoble - Rhône-Alpes 655, avenue de l'Europe, 38334 Montbonnot Saint Ismier Téléphone : +33476615200-Télécopie +33476615252 


\section{Capacité de réseaux maillés sans fil: éléments déterminants et caractères insensibles}

Résumé : La capacité d'un réseau reflète la charge de trafic qu'il peut écouler. C'est un facteur prépondérant de la qualité de service, en particulier dans le contexte très contraint des réseaux radio maillés 802.11. Dans cet article, nous considérons deux visions complémentaires de la capacité. Une capacité globale définie comme étant la somme des trafics sortants du réseau, et une capacité par flux mettant en évidence l'iniquité entre les flux dans le réseau. Nous étudions le comportement de ces capacités face à plusieurs paramètres : protocoles de routage, nombre et emplacement des passerelles reliant le réseau à l'Internet et régularité de la topologie physique du réseau. En se basant sur un large éventail de simulations, nous montrons le caractère insensible de la capacité à ces paramètres tout en soulignant qu'elle est directement liée à la congestion autour de la passerelle. De plus, nous montrons qu'en augmentant le nombre de passerelles, la capacité tend vers une borne maximale.

Mots-clés : Réseaux Radio Maillés, Capacité, Routage, Topologie. 


\section{Introduction}

Wireless mesh networks (WMNs) are proliferating as access network infrastructure [1] for broadband mobile services. In this paper, we consider a wireless mesh network based on collaboration between access points (a.k.a. routers) and organized in a twofold architecture as depicted in Fig. 1. The access points are equipped with a routing functionality and communicate together through radio links, forming a wireless backhaul infrastructure. Their role is, on the one hand, to collect the traffic generated by -potentially mobile- clients and, on the other hand, to forward these traffic through multi-hop communications toward some dedicated routers, called gateways, that bridge the backhauling network to the Internet.

Since the architecture is twofold, one can distinguish two domains of communications: one between clients and access points and another one among the routers of the access points. We assume that these two domains of communications do not interfere together because they independent radio channels. We focus in this paper on the performances of the backhauling infrastructure formed by the network of routers. The traffic considered in the backhauling infrastructure is the aggregated traffic of the clients collected at the access points. Each router of the network has hence to forward a given demand toward the gateways. The performance of the network is then evaluated with respect to the quality of service that it is possible to provide to these traffic flows.

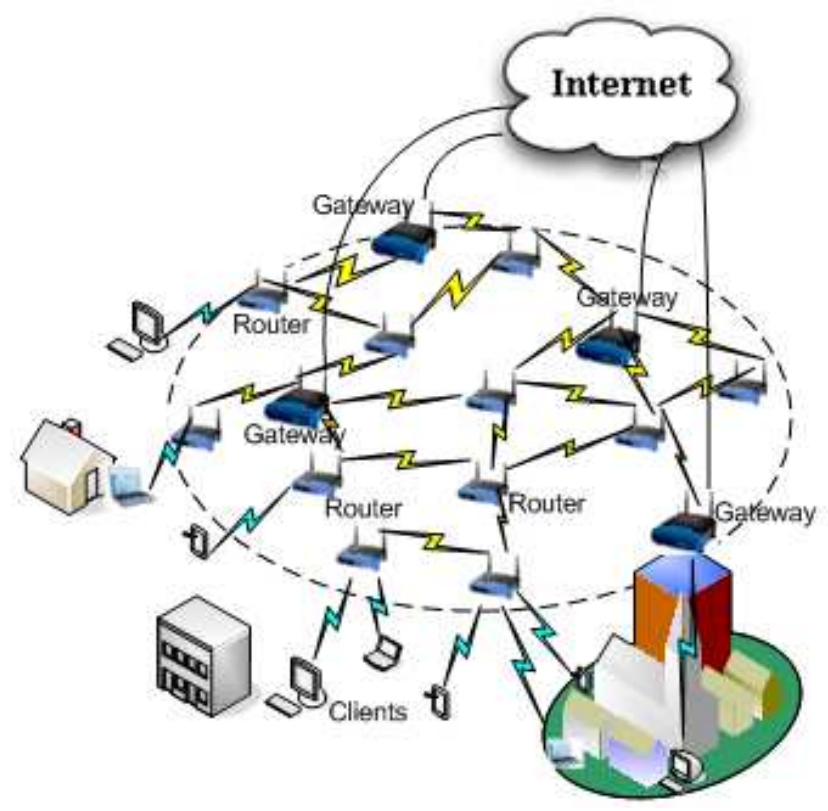

Figure 1: Wireless mesh network architecture: routers collect the traffic from clients (mobile or static) and forward it to the Internet through gateways.

In the generic settings of wireless networks, many paper of the literature have pointed out a critical behavior of the capacity $[2,3,4,5,6$. The capacity 
of a network is therefore a key issue of quality of service which is necessary to optimize. In this work, we aim at identifying the determining elements and insensible properties of the capacity of WMNs.

We consider two complementary definitions of the capacity. A first one, denoted network-wise capacity, is a measurement of the behavior of the whole network. It is defined as the sum of the traffics that have reached the gateways to the Internet. A second one, denoted flow-wise capacity, measures the capacity of each flow, that is the quantity of bandwidth allocated to the traffic collected by each router. To combine these two notions of capacity allows to highlight the unfairness among flows in network, which is a user-oriented point-of-view, within an operator-oriented look at the average behavior of the infrastructure.

Thorough simulations illustrate the behavior of these capacity notions face to several parameters. We show that the network-wise capacity as well as the flow-wise capacity are roughly insensitive to the choice of routing protocols and the placement of gateways in the network. We also show that the regularity of the network topology has little impact.

On the other hand, it is obvious that the addition of gateways improves the performances of the network in terms of capacity, latency, bandwidth allocated to each node... Nevertheless, we show that there is an upper bound under which the capacity keeps when the density of gateways in the network increases. The question of the impact of fairness on network performance is also addressed.

This article is organized as follows. A state of the art is presented in section 2 where we review the basic research that studied the radio network-wise capacity. Section 3 is dedicated to describing the methodology of our work, the assumptions made, and the radio model and routing protocols that we have used. Section 4 is devoted to the study of scenarios and analysis results. The last section concludes our work and presents the prospects and directions for the continuation of these researches.

\section{State of the art}

The capacity of a wireless mesh network is one of the most important criteria of quality of service. This metric is directly linked to the available bandwidth to each user of the network, or to the whole network.

Several notions of capacity have been defined in the literature. In [7], the capacity represents the quantity of flow that can be fairly split to the users. In $[2,3,6]$, the capacity is defined as the maximum bandwidth that can be allocated to each user. The study of the capacity may have different objectives. For an operator, the objective is to increase the number of served users while ensuring a better quality of service. For the user, improving the capacity is to obtain more bandwidth to increase his end-to-end flow.

The capacity of networks radio became a subject which interests a important number of research works. Several works focused on dedicated radio network topologies [2], confirmed by other studies [4,5], underline the highly sensitive behavior of the radio capacity. They showed that the capacity of a node in 
an ad-hoc wireless network decreases with $\frac{1}{\sqrt{n}}$ when the network cardinality, $\mathrm{n}$, increases. Unlike ad-hoc networks, wireless mesh networks are static and have bottlenecks located around the gateways. Therefore, the available capacity allocated to each node is reduced by a factor of $\frac{1}{n}[3]$. This result is found and extended in [8], which evaluates the difference of capacity provided by an ad-hoc network or an hybrid network, using linear programming models.

The capacity of a multi-hop mesh network is greater than the capacity of a single-hop. This is made possible by allowing spatial reuse, i.e., the activation of more than one link at a time [9]. Moreover, even with an optimal scheduling of calls, the network-wise capacity remains limited due to a bottleneck around the access point (gateway) 9,10 .

From these results, the researchers have focused their works on the study and optimization of the capacity. These works are based generally on optimization approaches, and heuristics. The optimization models, mainly using linear programming, have for objective to determine theoretical bounds to the capacity. In 10,11, a linear programming models of wireless mesh networks have been developed in order to compute a joint optimal resource allocation and routing. One of their main results is the identification of a critical area, located around the gateways, and characterized by an heavy congestion. A direct consequence on the gateways location optimization is a constraint on the distance separating the gateways that is enough to satisfy to provide the maximum capacity, while the routing outside the critical areas has little influence.

\section{Capacity evaluation methodology}

In all simulations, we consider a wireless mesh network using a single radio channel shared among all routers. These routers are assumed to be static.

The upload traffic in the network is uniformly distributed among the routers. We assume that the network operates in a saturated regime: each router periodically sends a given quantity of traffic which presents the aggregate traffic of its clients. This traffic is routed to gateways through multi-hop paths computed by the different routing protocols that are described in the following.

We assume that the communications in the infrastructure do not interfere with any other communication. We also assume that the interfaces connecting the gateways to the Internet have infinite capacity. Our study hence focuses on the behavior of the backhaul network.

The MAC layer used is a CSMA / CA with the classical Request to Send (RTS) / Clear to Send (CTS) mechanism [12].

We study two kinds of topologies. On the one hand, most measurements are achieved on grid shaped topologies while, on the other hand, the role played by the regularity of these topologies on the performances is evaluated using topologies generated by a $2 \mathrm{D}$-Poisson process.

\subsection{Radio Model}

In a wireless network, a radio link depends on several parameters. It mainly depends on the relative locations of the transmitter and the receiver. It also depends on other parameters of the physical layer such as modulation, shadow- 
ing, path loss, fading and interferences 13 .

To have more realistic scenarios, a physical layer taking into account the phenomenon of attenuation in free space and interference is simulated. When a node $\mathrm{E}$ transmits a signal with a transmission power $\mathrm{P} t$, the signal power at the reception is of the form $\mathrm{P} t * L\left(d_{i j}\right)$, where $\mathrm{d}_{i j}$ is the euclidean distance between the transmitter and receiver and $\mathrm{L}($.$) is the attenuation function, or path-loss.$

The interference model that is implemented takes into account the perturbations caused by the transmissions of all other nodes in the network. With all these parameters, it asseses if a packet is correctly received. A signal sent by a node $\mathrm{E}$ can be received at a receiver $\mathrm{R}$ if and only if the SINR (Signal to Interference and Noise Ratio) at the receiver is upper a threshold $\theta$. This model is defined as follows:

$$
S I N R=\frac{\mathrm{P} t * L\left(d_{i j}\right)}{\mathrm{W}+I(R)}>\theta
$$

where $\mathrm{W} \in \mathbb{R}^{+}$represents the thermal noise at the receiver and $\mathrm{I}(R)$ is the sum of the signals received from competing communication.

\subsection{Routing protocols}

We consider four routing protocols in order to route the upload traffic from the routers to the gateways.

\subsubsection{Shortest path routing}

This routing protocol is based on the Dijkstra algorithm 14. The goal is to find the shortest path in terms of hops between source and destination. The global knowledge of the whole topology is necessary and obtained using periodic control packets.

\subsubsection{Geographic routing protocol}

This routing protocol is based on the knowledge of geographic position for each node using GPS-like positioning. The main idea is to compare, at each hop, the euclidean distance between all neighbors and the destination, and choose to forward the packet to the closest neighbor 15 .

\subsubsection{Random routing protocol}

This routing is based on a random walk. It means that at each hop, the packet is forwarded to a randomly chosen neighbor. This protocol does not require the knowledge of the whole network, but only the neighborhood of each router using hello packets.

Two strategies are used to improve the behavior of this protocol: i) the packet is sent to the destination if it is a neighbor and ii) a packet is never routed to a node which has no other neighbor. 


\subsubsection{Static routing protocol}

With this routing protocol, all the paths between source node and destination are manually entered. This protocol does not require the use of control packets.

\subsection{Performance evaluation criteria}

\subsubsection{Network capacity}

In our work, the network-wise capacity is the quantity of traffic sent by all nodes $(\mathbb{N})$ and forwarded to the Internet through the gateways $(\mathbb{K})$ during the simulation period. It is a view of the global bandwidth of the network shared among all nodes.

This metric represents the maximum quantity of traffic that the network can transmit to the Internet. A better network-wise capacity is necessary for providing a better quality of service to a larger number of users.

This metric is calculated as follows.

$$
C_{\text {network }}=\frac{\sum_{k \in \mathbb{K}} \sum_{n \in \mathbb{N}} \mid \text { Received_packets }(n \rightarrow \mathrm{k}) \mid}{\text { Simulation_time }}
$$

This metric does not illustrate the unfairness problem in the network. A more detailed view is necessary for taking into account the bandwidth allocated to each flow.

\subsubsection{Flow capacity}

It is defined by the sum of traffics sent by a router and received by the gateway during the observation period. This metric illustrates the bandwidth consumed by each router in the network. Thus, it allows to study the problem of unfairness in the distribution of bandwidth amonog the flows. This is a key point of the quality of service. In fact, an operator must ensure a bandwidth acceptable for each node in the network. This metric is calculated as follows.

$$
C_{\text {flow(n) }}=\frac{\sum_{k \in \mathbb{K}} \mid \text { Received_packets }(n \rightarrow k) \mid}{\text { Simulation_time }}
$$

These two metrics are complementary because the first gives a global vision of the network while the secons gives a detailed view.

All the following results are obtained through intensive simulations using the WSNet simulator 16 and a confidence interval of $95 \%$ is guaranteed.

\subsection{Clustering algorithm}

To study the impact of the increase of the gateways density towards the capacity of mesh networks, we considered two scenarios.

In the first one, presented in the Fig 2(a) the network is divided into $N$ clusters. Each cluster contains a gateway. Subsequently, each node sends its traffic to the closest gateway. The second scenario, presented in $2(\mathrm{~b})$ is similar to the first but in this case, each node chosen randomly and uniformly one of the gateways and sends its traffic. 


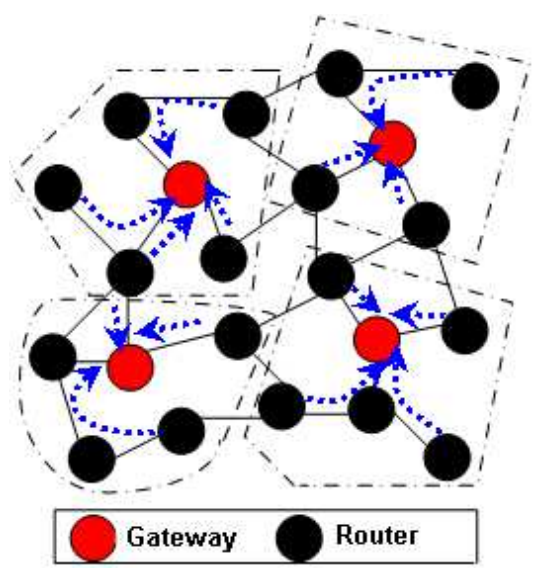

(a)

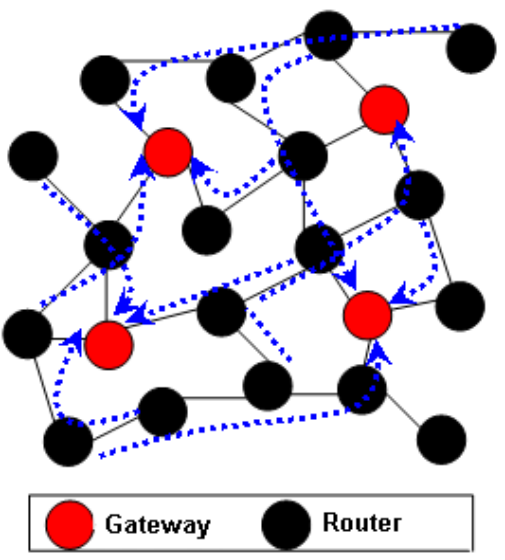

(b)

Figure 2: Scenarios of gateways sharing : (a) sending to the nearest gateway, (b) sending to a gateway chosen randomly

The difference between these two scenarios is that in the first one, if a client wants to connect to the Internet, then his traffic is routed to the nearest gateway. This is in order to reduce the number of hops (to the gateway), reduce the load on the links, reduce the impact of the collision domain, and thus to increase the throughput of each node 3 . But if a user wants to access a service not provided by the nearest gateway, or if it is busy or does not work, then his traffic must be routed to the appropriate gateway.

The construction of the clusters and the placement of each gateway in a cluster, are calculated by the algorithm 1. The objective is to group nodes in order to construct clusters with small diameter. Each cluster has almost the same cardinality. To do this, a node is selected then it combines its successive neighborhoods to get the cardinality desired (line 1-9). Then, in each cluster, the node closest to the barycenter is selected to be a gateway (line 10-14).

\section{RESULTS AND ANALYSIS}

We measure the evolution of network-wise capacity and flow-wise capacity face several parameters : routing protocols, network size, number and placement of gateways.

\subsection{The routing independence}

The independence of routing is highlighted by comparing the capacity obtained by the shortest paths routing, geographic routing, and the two types of random routing (classical and optimized) detailed in Part 3.2. Figures 3 and 4 show the evolution of network-wise capacity with network size in the case of grid topology and random topology, while Figures 5 and 6 show the flow-wise capacity.

We will see that there is no routing that provides a capacity significantly better than the others, only the random walk not optimized is less efficient. 

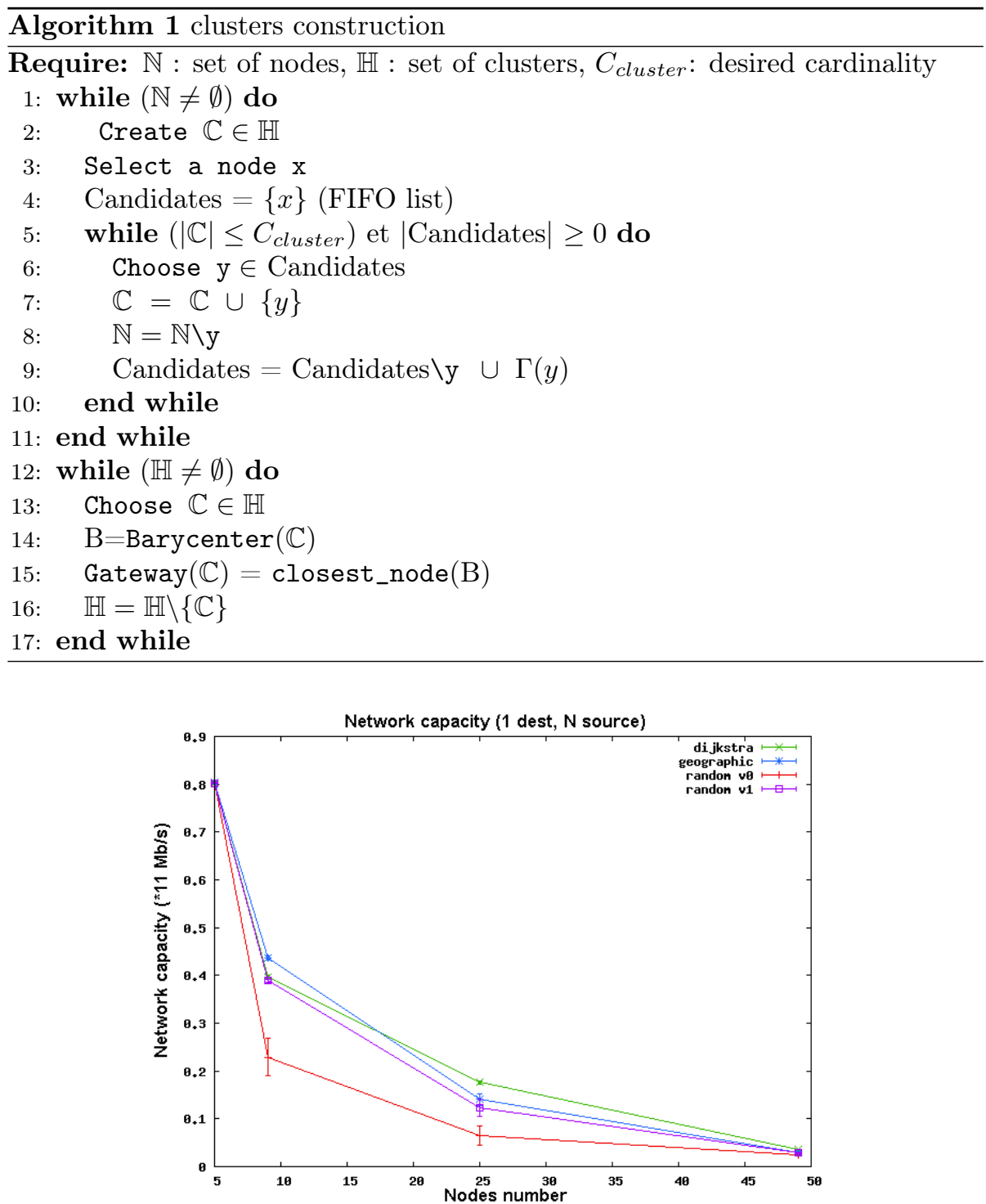

Figure 3: Network capacity vs routing and cardinality (grid)

For both types of topologies, the capacity evolves with the same way. We can note that the average capacity offered to nodes decreases more than $\frac{1}{n}$. This is due to the increase of collisions and interference where the network cardinality increases. Adding to all these factors, the presence of an important congestion around the gateway (bottleneck), the flow-wise capacity and the network-wise capacity decrease independently from routing protocols. In fact, when the network size increases, each node will have more neighbors in one and two hops. Thus, the access to the medium becomes increasingly difficult and the interference increases, especially around the gateway. 


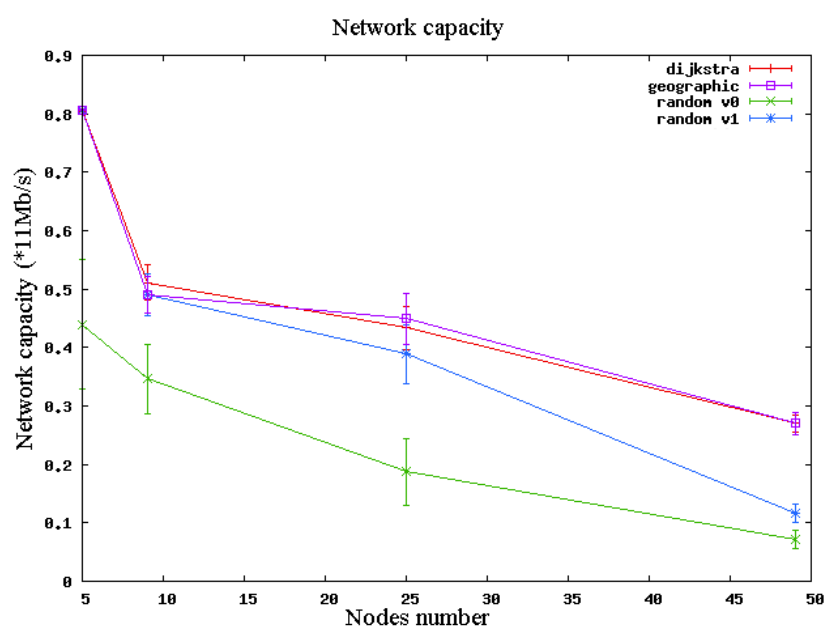

Figure 4: Network capacity vs routing and cardinality (random topology)

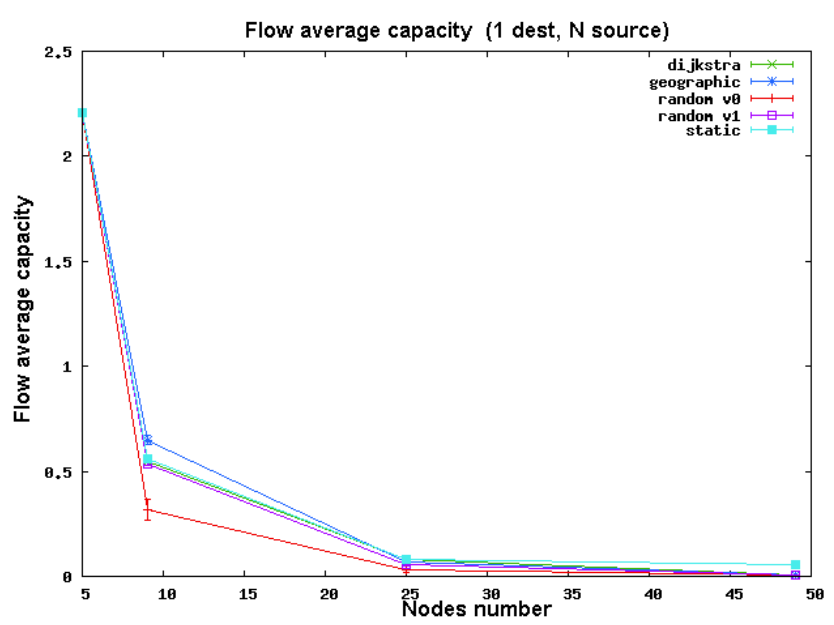

Figure 5: Flow capacity vs routing and cardinality (grid)

\subsection{Placement of gateways}

The small influence of the gateway placement is illustrated exhaustively on a $7 \times 7$ grid. Figure 7 illustrates the network-wise capacity obtained according to the location of the gateway. We find a classical result of axial symmetry of the grid because all the placements which are situated at the same distance of the grid center have the same capacity. We see that capacity increases slowly from the center to the board where the maximum capacity is reached. Indeed, the nodes that are situated at the center of the grid are characterized by a strong constraint caused by the congestion, interference and collision around these nodes, while nodes in the grid border have a more limited interference. 


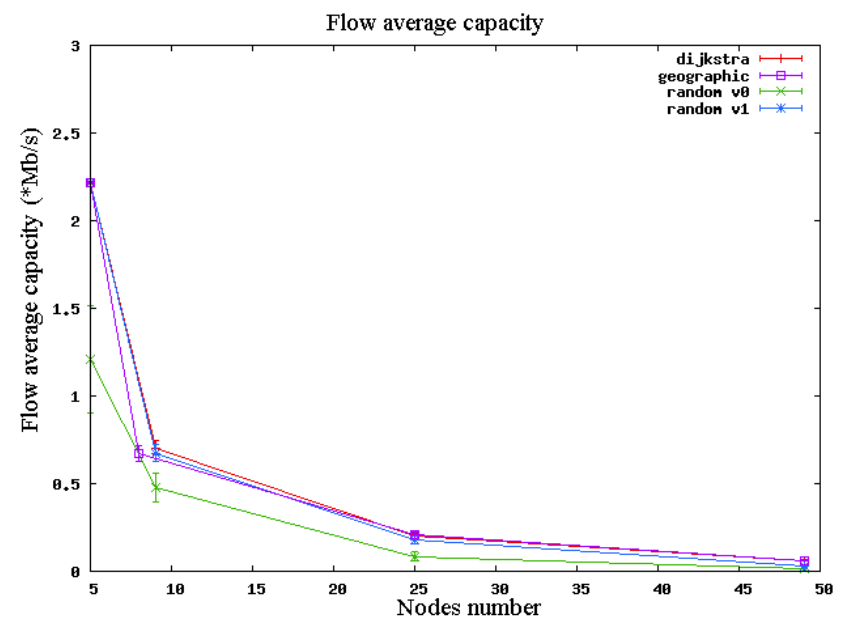

Figure 6: Flow capacity vs routing and cardinality (random topology)

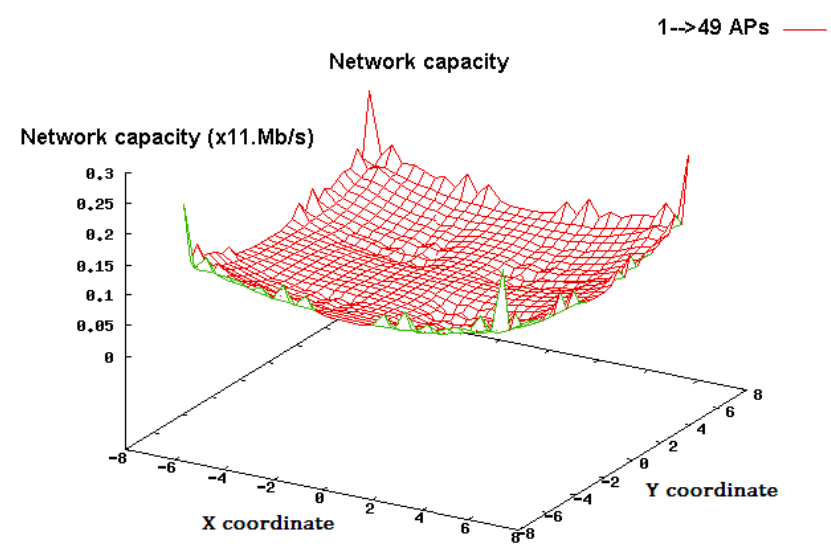

Figure 7: The global capacity of a grid according to the position of the gateway.

\subsection{The impact of the gateway density}

The impact of the gateway density in the network must be studied taking into account previous measurements. We study here two types of traffic : the first is sent to the nearest gateway (the barycenter of its cluster), the other is to gateway chosen randomly 3.4 .

Figure 8 shows the variation of network-wise capacity depending on the gateway density on a $12 \times 12$ grid. In the case of routing to the nearest gateway, the contribution of additional gateway is significant when their density is low, then it tends toward a threshold. The linear growth of the capacity is because the placement of the gateways does not matter as long as they are sufficiently spaced from each other gateway. When the interference zone of the gateways have a contact, then it becomes useless to add another. 
In the case of random gateway choice, network-wise capacity increases linearly with gateway density. Here, the distance (hops number) between a node and the gateway is more important which increases the collisions and interference on the multi-hops path. We tends towards an asymptotic behavior where the variation of network-wise capacity when a transition from $\mathrm{N}$ to $(\mathrm{N}+1)$ gateway results mainly of the traffic delivered directly to the Internet by the last added gateway.

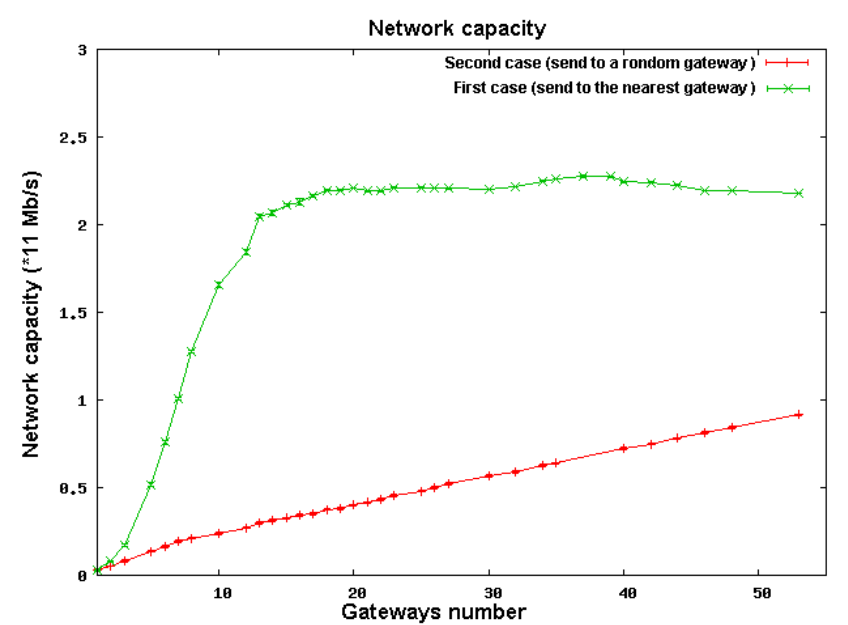

Figure 8: Network capacity vs gateways number (grid 12x12)

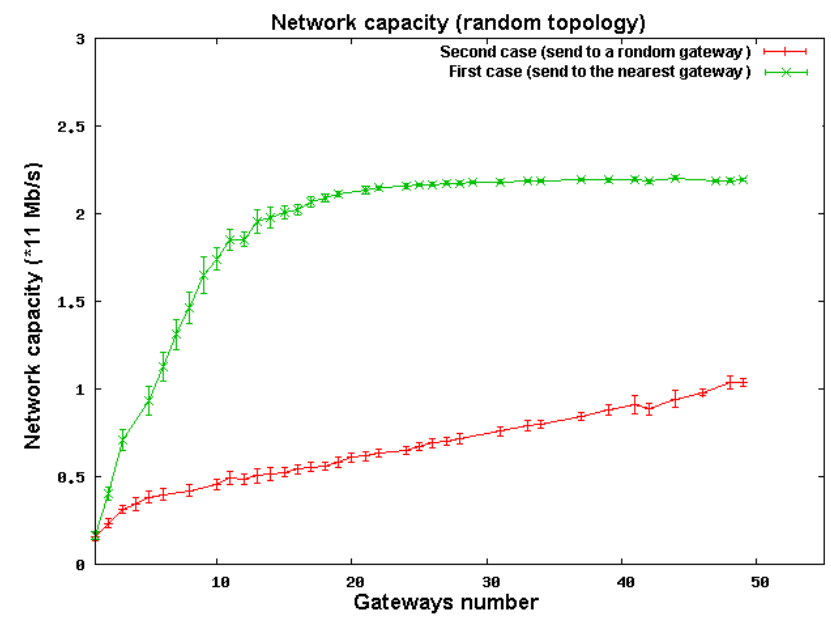

Figure 9: Network capacity vs gateways number (random topology)

\subsection{Independence of the topology}

The independence of the topology is illustrated by figure 9 where we show that the capacity of a random topology evolves similarly to that the grid for routing 


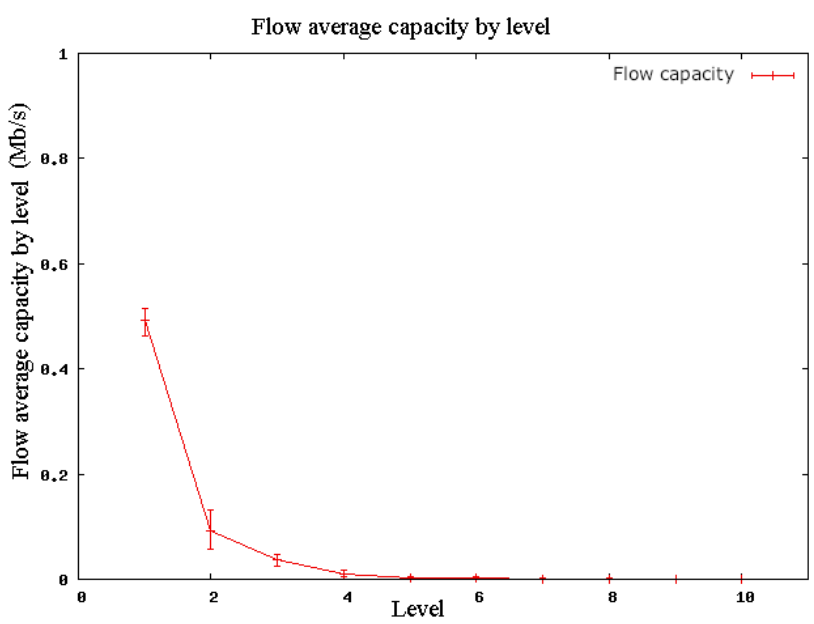

Figure 10: Flow average capacity by level (grid 11x11)

to the nearest gateway and to a random destination. We can see also this independence of topology by analyzing the figures presented in 4.1, where we found the same results for the grid topology and random topologies.

In this context, a result shows that under certain assumptions, a random network is a finite distance from a grid of equal density [17.

\subsection{Equity problem}

Several studies [18, 19] have highlighted some issues of equity in access to the radio medium that could occur with the use of 802.11 . The existence of this problem is confirmed by Figure 10 which shows the evolution of the average flow-wise capacity by level 1 . We see an inequality in the sharing of network bandwidth. Only the first nodes (especially the first) uses a large share of bandwidth, while very few packets of their successors reach the gateway. Most works on the network-wise capacity does not take into account this problem [3, 4, 5, 8, 6]. However, the unfairness problem has a big influence on network performance, in particular on the bandwidth allocated to each user. Also a mechanism, ensuring fairness in the radio network, risks to be very expensive in terms of traffic control and signaling, because it requires synchronization and a regulation of bandwidth consumption.

The question arises whether our results are strengthened or limited in the presence of a mechanism of equity.

\section{Conclusion}

In this work, we present results of simulations to isolate the determining elements of the capacity of wireless mesh network. We highlight some insensible properties of the capacity. We show that the regularity of the topology, the

\footnotetext{
${ }^{1}$ It's the set of nodes that have the same distance in terms of number of hops from the gateway.
} 
placement of gateways and the routing protocols have a limited impact on the network-wise and flow-wise capacity. These capacities are bounded to the bottlenecks around gateways. We show that the capacity tends toward a maximal value, when the number of gateways in the network increases. These results can be useful, in particular, during the designing of a wireless mesh network. Furthermore, these results moves us to optimize finely the behavior of the network in zones centered around the gateway.

\section{References}

[1] I. F. Akyildiz and X. Wang. A survey on wireless mesh networks. IEEE Radio Communications, vol. 43, no. 9:S23 - S30, September 2005.

[2] P. Gupta and P. R. Kumar. The capacity of wireless networks. IEEE Transactions on Information Theory, vol. 46:388-404, March 2000.

[3] J. Jun and M. L. Sichitiu. The nominal capacity of wireless mesh networks. IEEE Wireless Communications, vol. 10, no 5:p. 8-14, October 2003.

[4] M. Franceschetti, O. Dousse, D. Tse, and P. Tiran. Closing the gap in the capacity of random wireless networks. In IEEE International Symposium on Information Theory (ISIT), page 438, Chicago, June-July 2004.

[5] G. Méheut, S. Pérennes, and H. Rivano. Evaluation stochastique et simulation des réseaux radio. Research report 5989, 2006, INRIA.

[6] B. Liu, P. Thiran, and D. Towsley. Capacity of a wireless ad hoc network with infrastructure. MobiHoc, pages 239-246, Montreal, September 2007.

[7] C. Molle, F. Peix, and H. Rivano. Génération de colonnes pour le routage et l'ordonnancement dans les réseaux radio maillés. In CFIP, 2008.

[8] H. Rivano, F. Theoleyre, and F. Valois. Capacity evaluation framework and validation of self-organized routing schemes. In Workshop on Wireless Ad-hoc and Sensor Networks 2006. IWWAN'06. IEEE Communications Society on, volume 3, pages 779-785, 28-28 Sept. 2006.

[9] A. Girard, C. Rosenberg, and J. Luo. Engineering wireless mesh networks. In Proc. 19th IEEE International Symposium on Personal, Indoor and Mobile Radio Communications (PIMRC'08), Cannes, France, September 2008.

[10] C. Molle, F. Peix, S. Pérennes, and H. Rivano. Optimal routing and call scheduling in wireless mesh networks with localized information. In $C$. Kaklamanis and F. Nielson, editors, the fourth Symposium on Trustworthy Global Computing (TGC 2008), volume 5474 of LNCS:pages 171-185, Barcelona, Spain, November 2008.

[11] C. Gomes, S. Pérennes, and H. Rivano. Bottleneck analysis for routing and call scheduling in multi-hop wireless networks. In 4th IEEE Workshop on Broadband Wireless Access (BWA), New Orleans, US, December 2008. 
[12] IEEE Std 802.11-2007. Ieee standard for information technologytelecommunications and information exchange between systems- local and metropolitan area networks-specific requirements - part 11: Wireless lan medium access control (mac) and physical layer (phy) specifications. Technical report, June 2007.

[13] G. Chelius E. Ben Hamida and J-M Gorce. Impact of the physical layer modeling on the accuracy and scalability of wireless network simulation. In SCS SIMULATION 2009: Transactions of The Society for Modeling and Simulation International, 85:574-588, September 2009.

[14] Edsger. W. Dijkstra. A note on two problems in connexion with graphs. Numerische Mathematik, 1:269-271, 1959.

[15] B. Karp and H.T Kung. Greedy perimeter stateless routing for wireless networks. in Proceedings of the Sixth Annual ACM/IEEE International Conference on Mobile Computing and Networking (MobiCom 2000), pages 243-254, Boston, MA, August 2000.

[16] E. Ben Hamida, G. Chelius, and J-M Gorce. Scalable versus accurate physical layer modeling in wireless network simulations. In 22nd ACM/IEEE/SCS Workshop on Principles of Advanced and Distributed Simulation, pages 127-134, Italy, June 2008.

[17] R. Klasing, Z. Lotker, A. Navarra, and S. Pérennes. From balls and bins to points and vertices. Proceedings of the 16th Annual International Symposium on Algorithms and Computation (ISAAC 2005), 3827:757-766, December 2005.

[18] T. Nandagopal, T. Kim, X. Gao, and V. Bharghavan. Achieving mac layer fairness in wireless packet networks. In In Proceedings of the ACM Mobicom, pages 87-98, Boston, MA, USA, August 2000.

[19] V. Bharghavan, A. J. Demers, S. Shenker, and L. Zhang. Macaw : A media access protocol for wireless lan's. In SIGCOMM '94: Proceedings of the conference on Communications architectures, protocols and applications, pages 212-225, New York, NY, USA, 1994. 


\section{Contents}

$\begin{array}{lll}1 & \text { Introduction } & 3\end{array}$

\begin{tabular}{|lll}
2 & State of the art & 4
\end{tabular}

3 Capacity evaluation methodology 5

3.1 Radio Model . . . . . . . . . . . . . . . . . . . . . . . . . . . 5

3.2 Routing protocols $\ldots \ldots \ldots \ldots \ldots \ldots$

$3.2 .1 \quad$ Shortest path routing . . . . . . . . . . . . . 6

3.2 .2 Geographic routing protocol . . . . . . . . . . . . . . 6

3.2.3 Random routing protocol $\ldots \ldots \ldots \ldots$. . . . . . . . . 6

$3.2 .4 \quad$ Static routing protocol $\ldots \ldots \ldots \ldots \ldots$

3.3 Performance evaluation criteria $\ldots \ldots \ldots \ldots \ldots$

$3.3 .1 \quad$ Network capacity . . . . . . . . . . . . . . . . . . . 7

3.3 .2 Flow capacity $\ldots \ldots \ldots \ldots \ldots$

$3.4 \quad$ Clustering algorithm $\ldots \ldots \ldots \ldots \ldots \ldots \ldots$

$\begin{array}{llll}4 & \text { RESULTS AND ANALYSIS } & 8\end{array}$

4.1 The routing independence . . . . . . . . . . . . . . . . 8

4.2 Placement of gateways $\ldots \ldots \ldots \ldots$

4.3 The impact of the gateway density . . . . . . . . . . . . . 11

4.4 Independence of the topology $\ldots \ldots \ldots \ldots \ldots$

$4.5 \quad$ Equity problem $\ldots \ldots \ldots \ldots \ldots \ldots \ldots$

$\begin{array}{lll}5 \text { Conclusion } & 13\end{array}$ 
Centre de recherche INRIA Grenoble - Rhône-Alpes 655, avenue de l'Europe - 38334 Montbonnot Saint-Ismier (France)

Centre de recherche INRIA Bordeaux - Sud Ouest : Domaine Universitaire - 351, cours de la Libération - 33405 Talence Cedex Centre de recherche INRIA Lille - Nord Europe : Parc Scientifique de la Haute Borne - 40, avenue Halley - 59650 Villeneuve d'Ascq Centre de recherche INRIA Nancy - Grand Est : LORIA, Technopôle de Nancy-Brabois - Campus scientifique 615, rue du Jardin Botanique - BP 101 - 54602 Villers-lès-Nancy Cedex

Centre de recherche INRIA Paris - Rocquencourt : Domaine de Voluceau - Rocquencourt - BP 105 - 78153 Le Chesnay Cedex

Centre de recherche INRIA Rennes - Bretagne Atlantique : IRISA, Campus universitaire de Beaulieu - 35042 Rennes Cedex Centre de recherche INRIA Saclay - Île-de-France : Parc Orsay Université - ZAC des Vignes : 4, rue Jacques Monod - 91893 Orsay Cedex

Centre de recherche INRIA Sophia Antipolis - Méditerranée : 2004, route des Lucioles - BP 93 - 06902 Sophia Antipolis Cedex 